\title{
Time Delay Correlations in Chaotic Scattering: Random Matrix Approach
}

\author{
N. Lehmann ${ }^{1}$, D.V. Savin ${ }^{2}$, V.V. Sokolov ${ }^{2}$, H.-J. Sommers ${ }^{1}$ \\ ${ }^{1}$ Fachbereich Physik, Universität Gesamthochschule Essen, D-4300 Essen, Germany, \\ ${ }^{2}$ Budker Institute of Nuclear Physics, 630090 Novosibirsk, Russia
}

\begin{abstract}
We study the correlations of time delays in a model of chaotic resonance scattering based on the random matrix approach. Analytical formulae which are valid for arbitrary number of open channels and arbitrary coupling strength between resonances and channels are obtained by the supersymmetry method. We demonstrate that the time delay correlation function, though being not a Lorentzian, is characterized, similar to that of the scattering matrix, by the gap between the cloud of complex poles of the $S$-matrix and the real energy axis.
\end{abstract}

\section{Introduction}

The duration of particle collisions is an interesting and important aspect of general scattering theory which is in a sense complementary to the energy representation ordinarily used. A collision is characterized in this case by the time delay of particles in the region of interaction. Wigner [1] was the first who considered the time on which a monochromatic particle with given angular momentum is delayed during its elastic scattering. He established the connection of this time delay to the energy derivative of the scattering phase shift. The sharper the energy dependence of the phase shift is the longer is the time delay.

Later on Smith [2] extended the time delay concept on many channel problems introducing the time delay matrix

$$
Q^{a b}(E)=-i \hbar\left\{\frac{d}{d \varepsilon} \sum_{c} S^{a c}\left(E+\frac{\varepsilon}{2}\right) S^{* c b}\left(E-\frac{\varepsilon}{2}\right)\right\}_{\varepsilon=0}
$$

in the channel space. Here $S$ is the scattering matrix and the summation index $c$ runs over all the $M$ open scattering channels. The matrix $Q$ is hermitian; its diagonal element $Q^{c c}$ coincides with the mean duration of collision (time delay) in the $c$-th entrance channel. 
Generally speaking, the delays are different in different channels $c$. Taking the trace of the Smith matrix, one arrives at the simple weighted-mean characteristic

$$
Q(E)=\frac{1}{M} \sum_{c} Q^{c c}=-\frac{i}{M} \frac{d}{d E} \ln \operatorname{det} S(E)
$$

of the duration of collisions. Eq. (2) is just the many-channel version of the well-known simple Wigner formula. (Here and below we set $\hbar=1$.)

The time delay turns out to be an especially pertinent concept for the chaotic resonance scattering encountered in atomic, molecular and nuclear physics [3, [1], as well as in the scattering of electromagnetic microwaves [5, 6, 7] in resonance billiard-like cavities. The quantity $Q(E)$, being closely connected to the complex energy spectrum of resonance states, shows in its energy dependence strong fluctuations around a smooth regular variation. The two kinds of variation on different energy scales are naturally decomposed

$$
Q(E)=\langle Q(E)\rangle+Q_{f l}(E)
$$

with an energy or ensemble averaging. By this, the slow energy dependence of time delay is revealed whereas the two-point autocorrelation function

$$
C_{Q}(E, \varepsilon)=\left\langle Q_{f l}\left(E+\frac{\varepsilon}{2}\right) Q_{f l}\left(E-\frac{\varepsilon}{2}\right)\right\rangle=\left\langle Q\left(E+\frac{\varepsilon}{2}\right) Q\left(E-\frac{\varepsilon}{2}\right)\right\rangle-\left\langle Q\left(E+\frac{\varepsilon}{2}\right)\right\rangle\left\langle Q\left(E-\frac{\varepsilon}{2}\right)\right\rangle
$$

is used to characterize the time delay fluctuations.

To the best of our knowledge, the first consideration of these fluctuations has been made numerically as well as analytically in [8] and [9] in the framework of rather peculiar model of resonance elastic quantum scattering on a leaky surface of constant negative curvature. The noteworthy feature of this model is that the poles of the scattering amplitude turn out to correspond to zeros of the famous Riemann $\zeta$-function. The real parts of the poles are therefore supposed [10] to be chaotically distributed similar to the eigenvalues of the Gaussian Unitary Ensemble whereas all their imaginary parts (the widths of resonances) are the same. The latter very specific property partly deprives the model of its interest since actual single-channel widths are known to exhibit quite large fluctuations 11.

The width fluctuations are suppressed when many channels are open. In this case semiclassical approximation can be as a rule expected to be valid. The semiclassical analysis of the time delay in terms of closed periodic orbits is given in [12]. It is in particular emphasized there that only the tail of the correlation function (4) corresponding to the very large values of $\varepsilon$ can immediately be related to the (short) periodic orbits. Quite opposite, the central peak near the point $\varepsilon=0$ is formed as a result of a strong interference of many orbits. Therefore, its width describing the long-time asymptotic behaviour of the Fourier transform has no direct connection to the classical escape rate and has rather to be calculated on the pure quantum ground. This is in line with the results of the analysis [13] of distribution of the resonance widths in the three discs scattering problem.

It is now generally acknowledged that the random matrix theory [14 represents a suitable and reliable foundation for description of local properties of dynamical quantum 
chaos [15]. We therefore use below a random matrix model of chaotic scattering to calculate the time delay autocorrelation function. We suppose as usual that the number $N$ of resonances is asymptotically large and use the powerful supersymmetry technique [16] first applied to chaotic scattering problems in [17]. The number $M$ of the (statistically equivalent) scattering channels can be small or large or can even scale with the number of resonance states. One can treat the latter two cases [4, 18, 19] as a "semiclassical limit" in the matrix model. We show here that the time-delay local fluctuations are governed, similar to those of the $S$-matrix [19], by the gap between the real axis and the upper edge of the distribution of resonance energies in the complex energy plane. We compare this result with that obtained in the framework of the periodic orbit approach.

In the next section our statistical matrix model is briefly presented. The connections of average time delay with the resonance spectrum and S-matrix fluctuations are elucidated in sec. 3. After a short description in sec. 4 of the supersymmetry method which we use the main analytical results for the time delay correlation function are given and discussed in detail in sec. 5. Some numerical results shedding additional light upon properties of the time delay correlations are gathered in sec. 6 . We close with a brief summary in sec. 7 .

\section{The Resonance Matrix Model}

According to the general scattering theory, the evolution of the $N$-level unstable system formed on intermediate stage of a resonance collision is described [20, 21, 22] by the effective Hamiltonian

$$
\mathcal{H}=H-i \gamma W, \quad W=V V^{T} .
$$

The Hamiltonian (5) acts within the intrinsic $N$-dimensional space but acquires, due to the elimination of continuum variables, an antihermitian part. The hermitian matrix $H$ is the internal Hamiltonian with a discrete spectrum whereas the rectangular $N \times M$ matrix $V$ consists of the amplitudes $V_{m}^{c}$ of transitions between $N$ internal and $M$ channel states. These amplitudes are real in T-invariant theory, so that the matrix $W$, similar to $H$, is real and symmetric. As usual, we neglect the smooth energy dependence of $V$ and $W$. The dimensionless parameter $\gamma$ characterizes the strength of the coupling of the internal motion to the continuum.

The poles of the resonance scattering matrix in the complex energy plane are those of the Green's function [20, 21, 22]

$$
\mathcal{G}(E)=(E-\mathcal{H})^{-1} .
$$

They coincide with the eigenvalues $\mathcal{E}_{n}=E_{n}-\frac{i}{2} \Gamma_{n}$ of the effective Hamiltonian $\mathcal{H}$ with $E_{n}$ and $\Gamma_{n}$ being the energy and width of $n$-th resonance state. It what follows, the properties of the spectrum of complex energies $\mathcal{E}_{n}$ play the crucial role.

The intrinsic chaoticity of the internal motion of long-lived intermediate system manifests itself by chaotic fluctuations in resonance scattering and demands a statistical consideration. Therefore the random matrix approach extending the well-known [11, 14] 
description of chaotic bounded systems has been worked out in [23, 17, 22]. It is usually assumed that the hermitian part $H$ of the effective Hamiltonian belongs to the Gaussian Orthogonal Ensemble (GOE),

$$
\left\langle H_{n m}\right\rangle=0, \quad\left\langle H_{n m} H_{n^{\prime} m^{\prime}}\right\rangle=\frac{\lambda^{2}}{N}\left(\delta_{n n^{\prime}} \delta_{m m^{\prime}}+\delta_{n m^{\prime}} \delta_{m n^{\prime}}\right)
$$

In the limit $N \rightarrow \infty$ eigenvalues of $H$ are situated in the interval $[-2 \lambda, 2 \lambda]$ with the density given by Wigner's semicircle law. Following [22], we suggest the transition amplitudes $V_{n}^{c}$ also to be statistically independent Gaussian variables,

$$
\left\langle V_{n}^{a}\right\rangle=0,\left\langle V_{n}^{a} V_{m}^{b}\right\rangle=\frac{\lambda}{N} \delta^{a b} \delta_{n m} .
$$

We will use below the ensemble (17,8) to calculate the average quantities defined in (3, 4).

\section{Time Delay and Resonance Spectrum}

Since we have neglected a smooth energy dependence of the effective Hamiltonian (5), the poles $\mathcal{E}_{n}$ in the lower part of the complex energy plane are the only singularities of the resonance scattering matrix. Due to the unitarity condition their complex conjugates $\mathcal{E}_{n}^{*}$ serve as $S$-matrix's zeros. These two conditions result in the representation

$$
\operatorname{det} S(E)=\prod_{n} \frac{E-\mathcal{E}_{n}^{*}}{E-\mathcal{E}_{n}} .
$$

Substituting eq.(9) in eq.(2), we come to the important connection

$$
Q(E)=-2 \operatorname{Im}\left\{\frac{1}{M} \operatorname{tr} \mathcal{G}(\mathcal{E})\right\}=\frac{1}{M} \sum_{n} \frac{\Gamma_{n}}{\left(E-E_{n}\right)^{2}+\frac{1}{4} \Gamma_{n}^{2}}
$$

between the time delay and the trace of the Green's function (6) of the intermediate unstable system. The time delay is entirely determined by the spectrum of complex energies of this system. The collision duration directly reflects the statistical properties of resonances. This is in contrast to the scattering amplitudes $S^{c c^{\prime}}$ which explicitly depend also on the transition amplitudes $V_{n}^{c}$.

The ensemble averaging of eq.(10) gives

$$
\langle Q(E)\rangle=\frac{2}{m \lambda} \operatorname{Re} g(E)
$$

where $m<1$ is the ratio $M / N$ and the function

$$
g(E)=i \lambda \frac{1}{N}\langle\operatorname{tr} \mathcal{G}(E)\rangle
$$

satisfies the cubic equation [19]

$$
g(E)-\frac{1}{g(E)}+\frac{m \gamma}{1+\gamma g(E)}-i \frac{E}{\lambda}=0 .
$$


The (unique) solution with a positive real part has to be chosen. It can be seen from the consideration given in 19 that this real part is close to $\frac{\lambda}{N} \pi \rho(E)$ with $\rho(E)$ being the projection on the real energy axis near the scattering energy $E$ of the density of resonance levels in the complex energy plane.

On the other hand, averaging eq.(II) directly, we express $\langle Q\rangle$ in terms of the two-point $S$-matrix correlation function [17, 19]. In the limit of a large number of statistically equivalent channels, $M \gg 1$, scaling with the number of resonances $N$

$$
\langle Q\rangle=-\left.i \frac{d C_{S}(\varepsilon)}{d \varepsilon}\right|_{\varepsilon=0}+\left.i \frac{d \mathcal{T}(\varepsilon)}{d \varepsilon}\right|_{\varepsilon=0} .
$$

Here 19

$$
C_{S}(\varepsilon)=\frac{i \Gamma(\varepsilon)}{\varepsilon+i \Gamma(\varepsilon)} \mathcal{T}(\varepsilon) \equiv K(\varepsilon) \mathcal{T}(\varepsilon)
$$

with the two smooth functions defined by

$$
\Gamma(\varepsilon)=\frac{m}{2} \lambda \frac{\mathcal{T}(\varepsilon)}{g(\varepsilon / 2)} \quad, \quad \mathcal{T}(\varepsilon)=\frac{4 \gamma g(\varepsilon / 2)}{[1+\gamma g(\varepsilon / 2)]^{2}}
$$

and we set $E=0$ for the sake of simplicity. The quantity

$$
C_{S}(0)=\mathcal{T}(0) \equiv T
$$

coincides with the transmission coefficient $T=1-|\langle S\rangle|^{2}$. With eq.(15) taken into account we obtain from (14)

$$
\langle Q\rangle=-\left.i T \frac{d K(\varepsilon)}{d \varepsilon}\right|_{\varepsilon=0}=\frac{T}{\Gamma_{0}},
$$

where we have designated $\Gamma(0)$ as $\Gamma_{0}$.

As long as the typical values of the quantity $\Gamma(\varepsilon)$ are small as compared to the parameter $\lambda$ characterizing the scale of the smooth $\varepsilon$-dependence of the function $\mathcal{T}(\varepsilon)$, the two factors on the r.h.s. of eq.(15) have quite different energy scales. Only the first fast varying factor $K(\varepsilon)$ describes the local fluctuations whereas the second one corresponds to the joint influence of all resonances giving rise to the processes with a very short duration. The latter came out from eq.(18). The average time delay of a non-monochromatic spatially small wave packet caused by the formation of a long-lived intermediate state [3, 26] is determined just by the factor $K(\varepsilon)$ [19

$$
\langle\tau\rangle=-\left.i \frac{d K(\varepsilon)}{d \varepsilon}\right|_{\varepsilon=0}=\Gamma_{0}^{-1} .
$$

This implies the connection [26, 19]

$$
\langle\tau\rangle=\langle Q\rangle / T=\frac{2 N}{\lambda M T} g(0) \approx \frac{2 \pi \rho}{M T} .
$$




\section{The Supersymmetry Method}

Now we calculate the correlation function (4). Taking into account the relation (10), one can cast eq.(4) into the form

$$
C_{Q}(E, \varepsilon)=\frac{2}{M^{2}} \operatorname{Re}\left\{\left\langle\operatorname{tr} \mathcal{G}\left(E+\frac{\varepsilon}{2}\right) \operatorname{tr} \mathcal{G}^{\dagger}\left(E-\frac{\varepsilon}{2}\right)\right\rangle-\left\langle\operatorname{tr} \mathcal{G}\left(E+\frac{\varepsilon}{2}\right)\right\rangle\left\langle\operatorname{tr} \mathcal{G}^{\dagger}\left(E-\frac{\varepsilon}{2}\right)\right\rangle\right\} .
$$

We also define the normalized quantity

$$
K_{Q}(E, \varepsilon)=\frac{C_{Q}(E, \varepsilon)}{\langle Q(E)\rangle^{2}} .
$$

The terms containing two Green's functions with poles at the same side from the real energy axis are omitted in (21). We will briefly return to this point later.

In the limit $\gamma=0$, when the system gets closed, the correlation function (21) becomes proportional to the GOE density-density correlation which consists [14] of the singular term $\delta(\pi \rho \varepsilon)$ and Dyson's smooth function $-Y_{2}(\pi \rho \varepsilon)$. Coupling to the continuum leads to appearing of a new energy scale caused by the decay processes. This scale is defined [19] by the quantity $\Gamma(\varepsilon)$ from eq.(16). One can anticipate a qualitative changing of the correlation function to occur on this scale. For larger distances the influence of the antihermitian part should fade away and the asymptotics of $C_{Q}$ for $\varepsilon \rightarrow \infty$ is expected to coincide with that of the Dyson's function $-Y_{2}(\pi \rho \varepsilon)$.

To perform the ensemble averaging in (21) we use the modification worked out in 19] of the supersymmetry technique [17]. Using the integral representation of Green's function as a multivariate Gaussian integral over commuting and anticommuting variables, one gains the possibility to accomplish the averaging exactly. With the help of the Fourier transformation in the supermatrix space the integration over initial auxiliary supervectors is then carried out. Going along this line, one finally arrives at

$$
\left\langle\operatorname{tr} \mathcal{G}\left(E+\frac{\varepsilon}{2}\right) \operatorname{tr} \mathcal{G}^{\dagger}\left(E-\frac{\varepsilon}{2}\right)\right\rangle=-\frac{N^{2}}{4}\left\langle\operatorname{str}\left(\sigma \eta_{1}\right) \operatorname{str}\left(\sigma \eta_{2}\right)\right\rangle_{\mathcal{L}}
$$

Here the shorthand $\langle\ldots\rangle_{\mathcal{L}}$ is used to denote the integral

$$
\langle\ldots\rangle_{\mathcal{L}}=\int d[\sigma] d[\hat{\sigma}] \exp \{-N \mathcal{L}(\sigma, \hat{\sigma})\}(\ldots)
$$

over two $8 \times 8$ supermatrices $\sigma$ and $\hat{\sigma}$ with the measure defined by the Lagrangian [19]

$$
\mathcal{L}(\sigma, \hat{\sigma})=\frac{1}{4} \operatorname{str} \sigma^{2}-\frac{i}{2} E \operatorname{str} \sigma-\frac{i}{2} \operatorname{str}(\sigma \hat{\sigma})+\frac{1}{2} \operatorname{str} \ln (\hat{\sigma})+\frac{m}{2} \operatorname{str} \ln (1+\gamma \sigma \eta)-\frac{i}{4} \varepsilon \operatorname{str}(\sigma \eta) .
$$

The diagonal supermatrices appearing above are equal to

$$
\begin{gathered}
\eta=\operatorname{diag}(1,1,-1,-1,1,1,-1,-1) \\
\eta_{1}=\operatorname{diag}(1,1,0,0,-1,-1,0,0) \quad \eta_{2}=\operatorname{diag}(0,0,1,1,0,0,-1,-1) .
\end{gathered}
$$


Here we have set the GOE parameter $\lambda$ equal to one.

The supermatrix $\sigma$ can be decomposed in the following way [17

$$
\sigma=T_{0} \sigma_{R} T_{0}^{-1}
$$

where $T_{0}$ is a transformation from a non-compact manifold whereas the matrix $\sigma_{R}$ is diagonalized by transformations from a compact one. This implies a corresponding decomposition of the integrals on the r.h.s. of (24)

$$
\langle\ldots\rangle_{\mathcal{L}}=\int \mathcal{F}\left(\sigma_{R}\right) d\left[\sigma_{R}\right] d[\hat{\sigma}] \exp \left\{-N \mathcal{L}_{R}\left(\sigma_{R}, \hat{\sigma}\right)\right\} \int d \mu \exp \left\{-N \mathcal{L}_{\mu}\left(\sigma_{R}, T_{0}\right)\right\}(\ldots) .
$$

The Berezinian $\mathcal{F}\left(\sigma_{R}\right)$ depends only on the eigenvalues of $\sigma_{R} ; d \mu$ is the invariant measure of the manifold of non-compact transformations $T_{0}$. At last, the Lagrangian (25) is splitted into two parts, $\mathcal{L}_{R}$ and $\mathcal{L}_{\mu}$, given by

$$
\begin{aligned}
& \mathcal{L}_{R}(\sigma, \hat{\sigma})=\frac{1}{4} \operatorname{str} \sigma_{R}^{2}-\frac{i}{2} E \operatorname{str} \sigma_{R}-\frac{i}{2} \operatorname{str}\left(\sigma_{R} \hat{\sigma}\right)+\frac{1}{2} \operatorname{str} \ln (\hat{\sigma}), \\
& \mathcal{L}_{\mu}\left(\sigma_{R}, T_{0}\right)=-\frac{i}{4} \varepsilon \operatorname{str}\left(\sigma_{R} T_{0}^{-1} \eta T_{0}\right)+\frac{m}{2} \operatorname{str} \ln \left(1+\gamma \sigma_{R} T_{0}^{-1} \eta T_{0}\right) .
\end{aligned}
$$

Only the second part $\mathcal{L}_{\mu}$ depends on the non-compact variables. The first one $\mathcal{L}_{R}$ is invariant under a transformation by $T_{0}$ since it is fully absorbed by an appropriate transformation of $\hat{\sigma}$. One can easily verify that the corresponding Berezinian is equal to unity.

Since the number of resonances $N \rightarrow \infty$, the integrations over $\sigma_{R}$ and $\hat{\sigma}$ can be carried out in the saddle-point approximation. At the same time, one has to integrate exactly over non-compact variables as long as the number of channels $M$ is finite $(m=0)$. The saddle-point approximation becomes valid for the latter integration when the number $M$ also tends to infinity ( $m$ is finite). We will consider both cases mentioned. To simplify formulae we restrict our further consideration to the center of the GOE spectrum $E=0$.

\section{Time Delay Correlation Function}

Let us first consider collisions with a fixed number of channels $M$. The logarithmic term in $\mathcal{L}_{\mu}$ being proportional to the small ratio $m$ does not influence then the saddle-point equations in the $\left(\sigma_{R}, \hat{\sigma}\right)$-sector. In particular, the term in (13) containing this ratio has to be omitted. The saddle-point equations are trivially solved in this case and at the point $E=0$

$$
\hat{\sigma}=-i \sigma_{R}^{-1} \quad, \quad \sigma_{R}=\eta .
$$

With integrations over $\sigma_{R}$ and $\hat{\sigma}$ being done, the correlation function (四) reduces to the integral

$$
K_{Q}(\varepsilon)=2 \operatorname{Re} \int d \mu \operatorname{str}\left(\kappa \alpha_{1}\right) \operatorname{str}\left(\kappa \alpha_{2}\right) \exp \left\{\frac{i}{2} \pi \rho \varepsilon \operatorname{str} \alpha_{1}-\frac{M}{2} \operatorname{str} \ln \left(1+\frac{1}{2} T \alpha_{1}\right)\right\}
$$


over the invariant measure of the non-compact manifold of $T_{0}$-matrices. Here $\alpha_{1,2}$ are the $4 \times 4$ supermatrices defined in [17], the supermatrix $\kappa=\operatorname{diag}(1,1,-1,-1)$ and

$$
T=\frac{4 \gamma}{(1+\gamma)^{2}}
$$

is the transmission coefficient (17) calculated in the limit of $m=0$.

The further calculations go along the line described in details in [17] and lead to the result

$$
\begin{gathered}
K_{Q}(\varepsilon)=\frac{1}{4} \int_{0}^{1} d \lambda_{0} \int_{0}^{\infty} d \lambda_{1} \int_{0}^{\infty} d \lambda_{2} \mu\left(\lambda_{0}, \lambda_{1}, \lambda_{2}\right)\left(2 \lambda_{0}+\lambda_{1}+\lambda_{2}\right)^{2} \cos \left\{\pi \rho \varepsilon\left(2 \lambda_{0}+\lambda_{1}+\lambda_{2}\right)\right\} \\
\times\left[\frac{\left(1-T \lambda_{0}\right)^{2}}{\left(1+T \lambda_{1}\right)\left(1+T \lambda_{2}\right)}\right]^{M / 2}
\end{gathered}
$$

where

$$
\mu\left(\lambda_{0}, \lambda_{1}, \lambda_{2}\right)=\frac{\left(1-\lambda_{0}\right) \lambda_{0}\left|\lambda_{1}-\lambda_{2}\right|}{\left[\left(1+\lambda_{1}\right) \lambda_{1}\left(1+\lambda_{2}\right) \lambda_{2}\right]^{1 / 2}\left(\lambda_{0}+\lambda_{1}\right)^{2}\left(\lambda_{0}+\lambda_{2}\right)^{2}} .
$$

The dependence of the function $K_{Q}$ on openness of the unstable system is fully contained in the last factor in (32). If at least one of the quantities $M$ or $T$ is equal to zero the threefold integral reduces to the single one [16]

$$
\begin{gathered}
K_{Q}^{(0)}(\varepsilon)=\int_{0}^{2} d t t\left(1-\frac{1}{2} \ln (t+1)\right) \cos (\pi \rho \varepsilon t)+\int_{2}^{\infty} d t\left(2-\frac{t}{2} \ln \frac{t+1}{t-1}\right) \cos (\pi \rho \varepsilon t) \\
=\delta(\pi \rho \varepsilon)-Y_{2}(\pi \rho \varepsilon)
\end{gathered}
$$

which is just the normalized GOE density-density correlation function.

Generally speaking, the threefold integral in (32) can be investigated for arbitrary number of channels $M$ only numerically using the methods developed in [24] (see the next section). However, this integral can be simplified if $M$ becomes large enough. Let the number $M$ grow still keeping the ratio $m=0$ and the product $M T=2 \pi \rho \Gamma_{W}$ (compare with (20)) fixed. The quantity $\Gamma_{W}$ is just the limiting value of $\Gamma_{0}$ with $T$ and $g$ calculated in the limit $m=0$. It coincides with the well-known semiclassical Weisskopf estimate 25] of the correlation length of Ericson fluctuations. Then

$$
\left[\frac{\left(1-T \lambda_{0}\right)^{2}}{\left(1+T \lambda_{1}\right)\left(1+T \lambda_{2}\right)}\right]^{M / 2} \rightarrow \exp \left\{-\pi \rho \Gamma_{W}\left(2 \lambda_{0}+\lambda_{1}+\lambda_{2}\right)\right\},
$$

and one obtains similar to eq.(33)

$$
K_{Q}(\varepsilon)=\int_{0}^{2} d t t e^{\left(-\pi \rho \Gamma_{W} t\right)}\left(1-\frac{1}{2} \ln (t+1)\right) \cos (\pi \rho \varepsilon t)
$$




$$
+\int_{2}^{\infty} d t e^{\left(-\pi \rho \Gamma_{W} t\right)}\left(2-\frac{t}{2} \ln \frac{t+1}{t-1}\right) \cos (\pi \rho \varepsilon t) .
$$

This is in close analogy with the consideration of the S-matrix correlation function made in [24].

A new convergency factor appeared in the integrals in (35) as compared to (33) where only the oscillating cosine cuts the integral in the region of asymptotically large $t$. This makes the function $K_{Q}$ finite for all values of $\varepsilon$ including zero, so that the $\delta$-function is now smeared out. The behaviour of $K_{Q}(\varepsilon)$ is quite different in the regions $\varepsilon \ll \Gamma_{W}$ and $\varepsilon \gg \Gamma_{W}$. In the first one it is determined by decays and therefore is sensitive to the coupling to the continuum. Quite opposite, for large $\varepsilon$ the behaviour becomes universal since the GOE fluctuations described by the Dyson's function $Y_{2}$ are restored. It is perfectly reasonable since an open system cannot be distinguished from a closed one during a small time $t \ll \Gamma_{W}^{-1}$.

The first $\gamma$-sensitive domain is widened when the width $\Gamma_{W}$ grows. In the case of small $\rho \Gamma_{W} \ll 1$ (isolated resonances) it is natural to set aside the contribution of asymptotics of the integrand presenting (35) in the form

$$
\begin{gathered}
K_{Q}(\varepsilon)=\frac{1}{\pi \rho} \frac{\Gamma_{W}}{\left(\varepsilon^{2}+\Gamma_{W}^{2}\right)}+ \\
\int_{0}^{2} d t e^{-\pi \rho \Gamma_{W} t}\left(t-\frac{t}{2} \ln (t+1)-1\right) \cos (\pi \rho \varepsilon t)+\int_{2}^{\infty} d t e^{-\pi \rho \Gamma_{W} t}\left(1-\frac{t}{2} \ln \frac{t+1}{t-1}\right) \cos (\pi \rho \varepsilon t) .
\end{gathered}
$$

The Lorentzian contribution with the width $\Gamma_{W}$ directly traced to the GOE $\delta$-function dominates in the domain $\varepsilon \lesssim \Gamma_{W}$. The sum of the integrals in the second line is negative for all values of $\varepsilon$ and approaches asymptotically the function $Y_{2}$ from above. We thus come to the conclusion that the correlation function vanishes at some intermediate point $\varepsilon_{0}$ which can be estimated as

$$
\varepsilon_{0} \simeq \sqrt{\frac{\Gamma_{W}}{\pi \rho}}
$$

using the condition

$$
\frac{1}{\pi \rho} \frac{\Gamma_{W}}{\left(\varepsilon_{0}^{2}+\Gamma_{W}^{2}\right)} \sim\left|Y_{2}\left(\rho \varepsilon_{0}\right)\right| \sim 1
$$

The regime of strongly overlapping resonances, $\rho \Gamma_{W} \gg 1$, is the most interesting. In this case the main contribution in $K_{Q}$ comes from the region of small $t$. Therefore, the second integral in (35) can be neglected. Dropping then out the small logarithmic term in the first integral and extending its upper limit to infinity, we arrive at

$$
K_{Q}(\varepsilon) \approx \int_{0}^{\infty} d t t e^{\left(-\pi \rho \Gamma_{W} t\right)} \cos (\pi \rho \varepsilon t)=\frac{1}{\pi^{2} \rho^{2}} \frac{\Gamma_{W}^{2}-\varepsilon^{2}}{\left(\varepsilon^{2}+\Gamma_{W}^{2}\right)^{2}}
$$

Corrections to this result are of higher order with respect to the parameter $\left(\rho \Gamma_{W}\right)^{-1}$. The function (38) is not a Lorentzian at all. Decreasing quadratically in a small vicinity of 
the point $\varepsilon=0$, it deviates subsequently from a Lorentzian, becomes zero at the point $\varepsilon=\Gamma_{W}$, reaches a negative minimum and approaches at last zero from below. Just the correlation function of such a form with $\Gamma_{W}$ substituted by the classical escape rate was conjectured in [12] as the limiting classical expression following from the periodic orbit picture. However, there is no room for the classical escape rate in the matrix models considered here. One can see that the found form has in fact quantum grounds.

One should return to the exact expressions $(27,28)$ if the ratio $m$ is finite. The resonances strongly overlap in this case. The saddle-point is now found to be

$$
T_{0}=1, \quad \hat{\sigma}=-i \sigma_{R}^{-1}, \sigma_{R}=g(\varepsilon / 2) \eta,
$$

where $g$ is the solution chosen in sec. 5 of the cubic equation (13). The sequential saddle-point integrations over $\sigma_{R}, \hat{\sigma}$ and then over the non-compact manifold result in the expression

$$
K_{Q}(\varepsilon)=-\frac{4}{M^{2} T^{2}} \operatorname{Re} \frac{\Gamma_{0}^{2}}{[\varepsilon+i \Gamma(\varepsilon)]^{2}}
$$

where the function $\Gamma(\varepsilon)$ defined in (16) is just the one appearing when the $S$-matrix fluctuations are considered [19].

The explicit dependence on $\varepsilon$ gives rise to a sharp variation of the correlation function (40) in the vicinity of zero if the typical values $|\Gamma(\varepsilon)| \ll 1$ (see eq.(15) and the discussion below). As long as the ratio $m$ is small, the quantity $\Gamma(\varepsilon)$ is small indeed and we can neglect its smooth $\varepsilon$-dependence for all $\varepsilon \lesssim \Gamma_{0} \approx \Gamma_{W}$. Eq.(40) is equivalent to eq. (38) within this domain. The asymptotic behaviour for large $\varepsilon$ also does not change since $\Gamma(\varepsilon)$ remains restricted for all $\varepsilon$. A small difference can appear only for intermediate values of $\varepsilon$.

However, for larger values of $m$ the deviation can become noticeable even near the point $\varepsilon=0$. In this case the next term in the power expansion

$$
\Gamma(\varepsilon) \approx \Gamma_{0}+\Gamma_{0}^{\prime} \varepsilon
$$

with respect to the smooth $\varepsilon$-dependence should be taken into account 19. Because of the smoothness, the derivative $\Gamma_{0}^{\prime}$ is small. One can see from eq.(13) that this derivative is pure imaginary. The form (38) is now reproduced again for sufficiently small $\varepsilon$,

$$
K_{Q}(\varepsilon)=\frac{4 \Gamma_{g}^{2}}{M^{2} T^{2}} \frac{\Gamma_{g}^{2}-\varepsilon^{2}}{\left(\varepsilon^{2}+\Gamma_{g}^{2}\right)^{2}},
$$

with

$$
\Gamma_{g}=\frac{\Gamma_{0}}{1+i \Gamma_{0}^{\prime}} .
$$

It has been proven in [19 that $\Gamma_{g}$, playing the role of the correlation length of the Ericson fluctuations, coincides with the gap between the distribution of resonance energies in the complex energy plane and the real energy axis. Therefore we come to the conclusion that the properties of fluctuations both of the $S$-matrix and time delay are described by the same quantity, the gap $\Gamma_{g}$, rather than the classical escape rate. 
Until now we neglected the "one-sided" contribution

$$
\begin{gathered}
\widetilde{C}_{Q}(\varepsilon)=\langle Q\rangle^{2} \widetilde{K}_{Q}(\varepsilon)= \\
-\frac{2}{M^{2}} \operatorname{Re}\left\{\left\langle\operatorname{tr} \mathcal{G}\left(\frac{\varepsilon}{2}\right) \operatorname{tr} \mathcal{G}\left(-\frac{\varepsilon}{2}\right)\right\rangle-\left\langle\operatorname{tr} \mathcal{G}\left(\frac{\varepsilon}{2}\right)\right\rangle\left\langle\operatorname{tr} \mathcal{G}\left(-\frac{\varepsilon}{2}\right)\right\rangle\right\}
\end{gathered}
$$

to the correlation function (4). As long as $m=0$, this contribution is of higher order in the parameter $N^{-1}$. However, this is not the case when the ratio $M / N$ is finite. So one has to calculate (44) explicitly. The well-known replica method [27] turns out to be sufficient for the latter purpose. Dropping here the corresponding rather cumbersome expressions we only note that the function $\widetilde{K}_{Q}(\varepsilon)$ is entirely expressed in terms of the slowly varying $g\left(\frac{\varepsilon}{2}\right)$ and varies slowly itself. It has got no pronounced resonance behaviour around the point $\varepsilon=0$ and constitutes a smooth background for the correlation function. Its value at the point $\varepsilon=0$ is approximately equal to

$$
\widetilde{K}_{Q}(0) \approx-\frac{1}{8 N^{2}}
$$

so that

$$
\left|\widetilde{K}_{Q}(0) / K_{Q}(0)\right| \approx \frac{1}{2}\left(\frac{\pi \rho \Gamma_{0}}{2 N}\right)^{2} .
$$

The ratio is small under the condition

$$
\pi \rho \Gamma_{0} \ll N \quad \text { or } \quad \Gamma_{0} \ll 1
$$

implying a clear-cut distinction of the local and global scales [19]. Such a scale separation is necessary for matrix models to be valid so far as the fluctuations are concerned.

The obtained form of the $\varepsilon$-dependence of the many-channel correlation function $C_{Q}$ is close to that found in [9] for the Gutzwiller's model of single-channel chaotic scattering on a space of negative curvature. The same values of all resonance widths and the outcoming possibility for resonances to overlap are two specific features of the model which are in fact in strong disagreement with properties of the resonance spectra represented by matrix models. In particular, the single-channel resonances cannot overlap at all in the latter models [22] and their widths fluctuate strongly. That is why our result for $M=1$ (see below) differs noticeably from the correlation function of ref.[9]. The situation changes when the number of channels is large. The width fluctuations diminish with the number $M$ of channels growing. Since the time delay depends, according to (10), only on properties of the complex energies of resonances and not on the number of channels directly, the correlation functions become similar in the two quite different cases compared.

It is worthy to note that the resonances overlapping strongly suppress the time delay fluctuations. Indeed, eq.(36) gives for isolated resonances

$$
K_{Q}(0)=\frac{1}{\pi \rho \Gamma_{W}} \gg 1
$$

whereas

$$
K_{Q}(0)=\frac{1}{\pi^{2} \rho^{2} \Gamma_{W}^{2}} \ll 1
$$

when they overlap. The duration of a collision thus becomes a good definite quantity in the "quasiclassical" limit. 


\section{Numerical results}

Excepting a few limiting cases considered above, further analytical study of (32) is not possible and one has to use numerical methods. However, the threefold integral as it stands does not suit for numerical computation. A very convenient substitution of the integration variables has been proposed in [24 to overcome all difficulties appearing. Following this author we reduce the expression (32) to the Fourier integral

$$
K_{Q}(\varepsilon)=\int_{0}^{\infty} d t F(t) \cos (\pi \rho \varepsilon t)
$$

with the Fourier transform $F(t)$ given by a double integral of a smooth function quite convenient for the numerical work. The asymptotic behaviour of $F(t)$ can be easily found explicitly

$$
F(t) \sim\left\{\begin{array}{ll}
t & \text { for } t \ll 1 \\
(1+T t)^{-M / 2} & \text { for } t \gg 1
\end{array} .\right.
$$

For a closed system $(T=0)$ the Fourier transform $F(t)$ tends to unity in the large$t$ asymptotics. This results in the $\delta$-term in the GOE density-density correlation. A singularity still survives even for an open system with one or two decay channels. The asymptotics (47) implies square root or logarithmic divergences correspondingly at the point $\varepsilon=0$ in these two cases.

In Fig. 1 the function $K_{Q}(x)$ versus $x=\rho \varepsilon$ is plotted for the case of a single open channel. The singular behaviour near zero as well as GOE-like asymptotics are shown. The dashed line represents the Dyson's function $-Y_{2}(\pi x)$. The calculation was made for the value $\gamma=1$; only some small domain around zero is sensitive to the choice of $\gamma$. The correlation function Fig.1 has little in common with that found in [9]. This discrepancy is due to the strong fluctuations of single-channel widths in our model in contrast to identical widths of all resonances in Gutzwiller's one.

For $M>2$ the quantity $K_{Q}(0)$ is finite and the correlation function approaches, as the number of channels grows, the asymptotics given by (35). The Fig. 2 demonstrates this for the ratio $K_{Q}(\varepsilon) / K_{Q}(0)$ in the case of overlapping resonances. In asymptotic regime (38) such a ratio is an universal function of the only variable $\varepsilon / \Gamma_{W}$. One can see how the exact result (32) gets more and more close to this universal behaviour.

The Lorentzian peak should dominate the ratio $K_{Q}(\varepsilon) / K_{Q}(0)$ in the domain $\varepsilon / \Gamma_{W} \lesssim$ $\left(\pi \rho \Gamma_{W}\right)^{-\frac{1}{2}} \gg 1$ when resonances are isolated (see (37)). Fig. 3 demonstrates this for two values of coupling constant $\gamma$.

As it has been mentioned above, the function $K_{Q}(\varepsilon)$ vanishes at some point $\varepsilon_{0}$. The position of this point as the function of the number of channels $M$ at several fixed values of $\gamma$ is shown in Fig. 4 for three different values of the coupling constant $\gamma$. It is clearly seen that the square root dependence for isolated resonances (see (37)) is replaced by the linear one for overlapping ones. 


\section{Summary.}

In this paper we have considered the fluctuations of the characteristic time of collisions in the framework of a random matrix model of resonance chaotic scattering. These fluctuations are entirely due to the fluctuations of the spectrum of complex resonance energies. We calculate analytically the time delay correlation function and investigate its properties analytically and numerically for different values of the number of channels and the strength of the coupling to the continuum. For any values of these parameters this function is far from being a Lorentzian. In particular, it vanishes at some point which plays the role of the characteristic correlation length of the fluctuations. In the "quasiclassical" limit of a large number of strongly overlapping resonances this length is given, similar to that of the S-matrix fluctuations, by the gap between the upper edge of the distribution of complex energies of resonances and the real energy axis. We do not expect that this quantity may be connected to the escape rate appearing in the classical theory of chaotic scattering. The latter has been conjectured in [28 to be the semiclassical limit for the correlation length in chaotic scattering.

\section{Acknowledgements}

We are grateful to F.Izrailev for his permanent interest to this work. Financial support by the Deutsche Forschungsgemeinschaft through the SFB 237 is acknowledged. For two of us (V.V.S. and D.V.S.) the research described in this publication was made possible in part by Grant No RB7000 from the International Science Foundation.

\section{References}

[1] E. Wigner, Phys. Rev. 98 (1955) 145.

[2] F.T. Smith, Phys. Rev. 118 (1960) 349; 119 (1960) 2098.

[3] V.L. Lyuboshitz, Phys. Lett. B 72 (1977) 41; Yad. Fiz. 27 (1978) 948 (Sov. Journ. Nucl. Phys. 27 (1978) 502); Pis.ZhETF 28 (1978) 32 (Sov. JETP Lett. 28 (1978) $30)$.

[4] C.H. Lewenkopf and H.A. Weidenmüller, Ann. of Phys.(N.Y.) 212 (1991) 53.

[5] S. Sridhar, Phys. Rev. Lett. 67 (1991) 785.

[6] H.-D. Gräf, H.L. Harney, H. Lengeler, C.H. Lewenkopf, C. Rangacharyulu, A.Richter, P. Schardt and H.A. Weidenmüller, Phys. Rev. Lett. 69 (1992) 1296.

[7] J. Stein and H.-J. Stöckmann, Phys. Rev. Lett. 68 (1992) 2867.

[8] D.M. Wardlaw and W. Jaworski, J. Phys. A: Math. Gen. 22 (1989) 3561.

[9] A. Shushin and D.M. Wardlaw, J. Phys. A: Math. Gen. 22 (1992) 1503. 
[10] H.L. Montgomery, Analytic Number Theory ed. H.G. Diamond Proc. Symp. Pure Math. 24 (Providence, RI: Am Math. Soc.)

[11] C.E. Porter (ed.) Statistical Theories of Spectra: Fluctuations, (Academic Press, New York, 1965).

[12] B. Eckhardt, Chaos 3 (1993) 613.

[13] P. Gaspard and S.A. Rice, J. Chem. Phys. 90 (1989) 2225, 2242,2255.

[14] M.L. Mehta, Random Matricies and the Statistical Theory of Energy Spectra, (N.Y., Acad. Press, 1967).

[15] O. Bohigas, M.-J. Giannoni, Chaotic Motion and and Random Matrix Theory, Lecture Notes in Phys., 209 (1984) 1; O. Bohigas, Random Matrix Theories and Chaotic Dynamics, Lecture Notes, Les Houches (North- Holland, Amsterdam, 1991).

[16] K.B. Efetov, Advan. in Phys. 32 (1983) 53.

[17] J.J.M. Verbaarschot, H.A. Weidenmüller and M.R. Zirnbauer, Phys. Rev. Lett. 52 (1984) 1597; Phys. Rep. 129 (1985) 367.

[18] F. Haake, F.M. Izrailev, N. Lehmann, D. Saher and H.-J. Sommers, Z. Phys. 88 (1992) 359.

[19] N. Lehmann, D. Saher, V.V. Sokolov and H.-J. Sommers, (to be published in Nucl. Phys. A).

[20] C. Mahaux and H.A. Weidenmüller, Shell-model Approach to Nuclear Reactions, (North-Holland, Amsterdam, 1969).

[21] I.Yu. Kobzarev, N.N. Nikolaev and L.B. Okun, Yad. Fiz. 10 (1969) 864; V.D. Kirilyuk, N.N. Nikolaev, L.B. Okun, ibid p.1081.

[22] V.V. Sokolov and V.G. Zelevinsky, Phys. Lett. B202 (1988) 140; Nucl. Phys. A504 (1989) 562.

[23] H.A. Weidenmüller, Ann. of Phys.(N.Y.) 158 (1984) 120.

[24] J.J.M. Verbaarschot, Ann. of Phys.(N.Y.) 168 (1986) 368.

[25] J.M. Blatt and V.F. Weisskopf, Theoretical Nuclear Physics, (Springer Verlag, N.Y., 1979).

[26] F.M. Dittes, H.L. Harney and A. Müller, Phys. Rev. A 45 (1992) 701; Ann. Phys. 220 (1992) 159.

[27] S.F. Edwards and P.W. Anderson, J. Phys. F 5 (1975) 965.

[28] U. Smilansky, The Classical and Quantum Theory of Chaotic Scattering, Lecture Notes, Les Houches (North-Holland, Amsterdam 1991). 


\section{Figures}

Fig. 1 The time delay correlation function (32) versus $x=\rho \varepsilon$ for $M=1$ and $\gamma=1.0$. The dotted curve is the Dyson's function $-Y_{2}(\pi x)$.

Fig. 2 Overlapped resonances. The normalized function $K_{Q}(\varepsilon) / K_{Q}(0)$ versus $x=\varepsilon / \Gamma_{W}$ for three values of $M=5,10$ and 20 (dash-dotted, dashed and dotted curves) and $\gamma=1.0$ The solid curve is the asymptotic expression (38).

Fig. 3 Isolated resonances. The normalized correlation function (36) for $\rho \Gamma_{W}=0.1$ and $\rho \Gamma_{W}=0.01$ (dashed and dotted curves), and Lorentzian (solid curve).

Fig. 4 The zero $\rho \varepsilon_{0}$ of $K_{Q}(\varepsilon)$ as function of $M$ for three coupling constants: $\gamma=0.01(\star)$ (in this case $\rho \varepsilon_{0}$ has been blown up by a factor 10), $\gamma=0.1(\bullet)$ and $\gamma=1.0(0)$. Solid and dashed lines are $\rho \Gamma_{W}$ and the dotted curve is the estimate (37). 